\title{
Factores de riesgo para tuberculosis pulmonar en trabajadores de salud. Hospital Nacional Dos de Mayo. Lima, Perú
}

\author{
Carlos Contreras Camarena* 1,a,b
}

RESUMEN

Objetivo: Determinar los factores de riesgo para tuberculosis pulmonar en trabajadores de salud del Hospital Nacional Dos de Mayo. Lima, Perú.

Materiales y métodos: Estudio analítico de casos y controles. La población de estudio fueron trabajadores de salud con diagnóstico de tuberculosis pulmonar del Hospital Nacional Dos de Mayo. Lima, Perú. Se recolectaron variables epidemiológicas (edad, sexo, comorbilidad, grupo ocupacional), variables ventilatorias (velocidad, dirección y patrón del flujo aéreo, sistema de ventilación cerrada) y ambientales (luz ultravioleta, aire acondicionado). Se utilizó análisis univariado, se determinó el riesgo relativo, estimado por Odds Ratio con su respectivo intervalo de confianza al $95 \%$. Posteriormente, se utilizó un modelo predictivo de regresión logística multivariado paso a paso para identificar los factores de riesgo. Se consideró el valor de $p<$ de 0,05 como significativo.

Resultados: La tasa de incidencia habitual de tuberculosis pulmonar fue de 348 por 100 000. Los factores asociados fueron velocidad del flujo aéreo menor de $0,7 \mathrm{~m} / \mathrm{s}$, presencia de aire acondicionado en los ambientes hospitalarios, elevado grado de hacinamiento (espacio libre menor de $2 \mathrm{~m}^{2} /$ persona), edad menor de 40 años, presencia de al menos una comorbilidad, y el tiempo de trabajo hospitalario menor de 20 años.

Conclusiones: La presencia de alto grado de hacinamiento y el tiempo de trabajo hospitalario menor a 20 años constituyen importantes factores de riesgo para tuberculosis pulmonar activa en trabajadores de salud del Hospital Nacional Dos de Mayo.

Palabras clave: Tuberculosis pulmonar; Trabajadores de la salud (Fuente: DeCS BIREME).

\section{Risk factors for pulmonary tuberculosis in health-care workers. Hospital Nacional Dos de Mayo. Lima, Peru}

\section{ABSTRACT}

Objective: To determine the risk factors for pulmonary tuberculosis in health-care workers of the Hospital Nacional Dos de Mayo in Lima, Peru.

Materials and methods: A case-control analytical study. The study population consisted of health-care workers of the Hospital Nacional Dos de Mayo in Lima, Peru, diagnosed with pulmonary tuberculosis. Epidemiological variables (age, sex, comorbidity, occupational group), ventilatory variables (airflow velocity, direction and pattern; closed ventilation system) and environmental variables (ultraviolet light, air conditioning) were collected. A univariate analysis was performed, and the relative risk was estimated by odds ratio and its corresponding $95 \%$ confidence interval. Subsequently, a multivariate logistic regression predictive model was used step by step to identify the risk factors. A p value $<0.05$ was considered meaningful.

Results: The usual incidence rate of pulmonary tuberculosis was 348 per 100,000 health-care workers. The associated factors were airflow velocity less than $0.7 \mathrm{~m} / \mathrm{s}$, use of air-conditioning in hospital rooms, high levels of overcrowding (available space less than $2 \mathrm{~m}^{2} /$ person), age less than 40 years, presence of at least one comorbidity, and hospital job time less than 20 years.

Conclusions: High levels of overcrowding and hospital job time less than 20 years are important risk factors for active pulmonary tuberculosis in health-care workers of the Hospital Nacional Dos de Mayo.

Keywords: Tuberculosis, pulmonary; Health personnel (Source: MeSH NLM).

1. Hospital Nacional Dos de Mayo. Lima, Perú.

a. Médico Internista.

b. Magister en Gerencia de Servicios Públicos de Salud.

* Autor corresponsal 


\section{INTRODUCCIÓN}

En nuestro país, se notifican aproximadamente $31 \mathrm{mil}$ casos nuevos de tuberculosis (TB) cada año. Para el 2016, la Organización Mundial de la salud (OMS), a través de su reporte anual, estimó una incidencia de tuberculosis de 117 por 100000 habitantes (90 - 148). Ese mismo año se notificaron 31079 casos de tuberculosis en el país, y el presupuesto nacional asignado para la tuberculosis fue de 101 millones de dólares americanos ${ }^{(1)}$.

La incidencia anual de tuberculosis en trabajadores de salud de diferentes países en vías de desarrollo fluctúa entre 25 a 5361 por 100 000. La magnitud del riesgo de infección varía según el tipo de establecimiento, grupo ocupacional, prevalencia de la tuberculosis en la población, proporción de pacientes hospitalarios que reciben atención y la eficacia de las medidas de control de TB en el hospital. La probabilidad de infectarse del personal de salud depende del grado y tiempo de contacto con el bacilo. Se han descrito ambientes hospitalarios donde existe mayor probabilidad de infectarse con el bacilo, como salas de emergencia, medicina y neumología. Así mismo, en los ambientes donde se realizan broncofibroscopía, cultivos de micobacterias, nebulización y necropsias. El riesgo de adquirir la enfermedad tiene una asociación evidente con la ocupación o profesión del personal de salud, especialmente, los trabajadores que permanecen mayor tiempo en contacto con los enfermos ${ }^{(2)}$.

La incidencia de tuberculosis en la población general ha descendido progresivamente desde la década del 90 hasta el 2003, a un ritmo de $8 \%$ anual, mientras que, a partir de esa fecha hasta el 2011 se ha mantenido estacionaria o presenta tendencia a aumentar, con una tasa entre 100 y 120 casos x 100000 habitantes, siendo Lima, el departamento de mayor incidencia de TB con 479,7/100 000 habitantes ${ }^{(3)}$.

A pesar de esta aparente disminución de la incidencia de la enfermedad, los casos de tuberculosis en los trabajadores de salud se han incrementado, especialmente, en los hospitales públicos pertenecientes al Ministerio de Salud. Este aumento de la incidencia de TB en los hospitales de la capital ha motivado la presente investigación.

El objetivo del estudio fue determinar los factores de riesgo para contraer tuberculosis pulmonar en personal de salud del Hospital Nacional Dos de Mayo.

\section{MATERIALES Y MÉTODOS}

Diseño y población de estudio

Se realizó una investigación analítica de casos y controles. Los casos fueron trabajadores del Hospital Nacional Dos de Mayo afectados de tuberculosis pulmonar durante el periodo de estudio. Los controles fueron trabajadores sin enfermedad tuberculosa quienes fueron pareados por categoría profesional, fueron seleccionados del padrón general de todos los empleados del hospital, se eligieron los respectivos controles por muestreo aleatorio sistemático, uno por cada caso. Fueron excluidos los casos con tuberculosis extrapulmonar, casos no registrados en la base de datos del médico del personal, casos y controles menores de 18 años y trabajadores de salud (TS), con permanencia menor a un mes en el servicio donde laboraba.

\section{Variables y mediciones}

Se consideraron las variables epidemiológicas (edad, genero, comorbilidad, grupo ocupacional, estatus laboral, tiempo de servicio), las variables ventilatorioambientales (velocidad, dirección y patrón del flujo aéreo, presencia de sistemas de ventilación cerrada, luz ultravioleta, aire acondicionado) y variables de medidas de hacinamiento.

El hacinamiento se calculó de acuerdo a la norma técnica de edificación de hospitales de tercer nivel de atención (NTS N¹19-MINSA/DGIEM-2015), y el Reglamento Nacional de Edificación del Perú, mediante su norma específica para Hospitales A.050 Salud ART 6. -RM 660_2014 MINSA_I art 6.2.1.16- (Tabla 1). Esta norma técnica recomienda un espacio de $12 \mathrm{~m}^{2}$ como espacio mínimo por cada cama en hospitalización en los servicios de Medicina Interna y Cirugía, de igual forma, dispone de $15 \mathrm{~m}^{2} /$ cama en los servicios de Ginecología, Emergencia y Cuidados Intensivos. Así mismo, recomienda áreas mínimas para triaje de $9 \mathrm{~m}^{2}$, y de $30 \mathrm{~m}^{2}$ para sala de espera en emergencia. Los ambientes de consultorio externo no deberán tener menos de 3 metros de ancho y el área mínima por cada ocupante será de $6 \mathrm{~m}^{2}$ por persona. El grado de hacinamiento en los diferentes ambientes hospitalarios se estableció teniendo en cuenta el área libre que cada TS dispone en su ambiente de trabajo, especialmente, en las horas de mayor demanda. Al área libre, se sustrajo el área que ocupaban los diferentes equipos, camas y bienes inmobiliarios. Se consideró hacinamiento inminente cuando el área libre fue menor o igual a $2 \mathrm{~m}^{2}$ por persona (disminución del $50 \%$ de área óptima recomendada). 
Tabla 1. Densidad de ocupación en zonas hospitalarias en función de la actividad. Reglamento Nacional de Edificación - Perú

\begin{tabular}{clc}
$\begin{array}{c}\text { Uso } \\
\text { previsto }\end{array}$ & \multicolumn{1}{c}{$\begin{array}{c}\text { Zona } \\
\text { (tipo de actividad) }\end{array}$} & $\begin{array}{c}\text { Ocupación } \\
\text { (m²/persona) }\end{array}$ \\
\hline \multirow{2}{*}{ Hospitalario } & Sala de espera & 0,8 \\
& Zona de hospitalización & 12 \\
& Área de servicios ambulatorio y diagnóstico & 10 \\
& Área de servicios auxiliares & 8 \\
& Oficinas administrativas & 10 \\
& Área de tratamiento de pacientes internos & 20 \\
\hline
\end{tabular}

Fuente: RNE A.050 SALUD ART 6. (RM 660_2014 MINSA_I art 6.2.1.16)

Se consideró flujo aéreo laminar cuando las ventanas y puertas tenían una disposición horizontal y opuestas, de manera que el flujo aéreo no encontraba obstáculo alguno a su paso por los ambientes y, más bien, ejercía un efecto de "barrido" ambiental. El flujo turbulento fue considerado cuando se encontraban obstáculos como paredes, muros, inmobiliario y otros enseres físicos al paso del aire. La velocidad del flujo aéreo se calculó con un minianemómetro marca UNI-T, modelo UT363.

Se midió la velocidad del flujo aéreo en puertas y ventanas $(\mathrm{m} / \mathrm{s})$, según correspondía a cada servicio y en diferentes horarios del día. Se determinó el promedio y se realizó el análisis univariado en los diferentes ambientes de riesgo.

La dirección del flujo aéreo se determinó usando una veleta. Se consideró apropiada si el flujo se dirigía desde sitios más limpios a áreas contaminadas, e inapropiada si el flujo se dirigía desde áreas contaminadas a áreas más limpias. Las otras variables menos complejas fueron dicotomizadas para el análisis univariado correspondiente.

\section{Análisis estadístico}

La información fue almacenada y procesada con el paquete estadístico de SSPS versión 23.0. Se utilizaron técnicas paramétricas y no paramétricas, análisis de tendencia central y análisis comparativos entre los casos y controles. La asociación de las variables estudiadas se determinó mediante análisis univariado con su respectiva razón de momios, consideradas como un estimado cercano del riesgo relativo. Se determinaron Odds Ratios (OR) con sus respectivos intervalos de confianza al $95 \%$. Se utilizó Chi-cuadrado cuando la frecuencia en las celdas de la tabla $2 \times 2$ fue mayor a cinco, y la prueba de Fisher cuando la frecuencia esperada en cualquier celda era menor de cinco. Se consideró el valor de $p<0,05$ como estadísticamente significativo. Las variables procesadas que mostraron un valor de $\mathrm{p}<0,05$ en el análisis univariado, fueron incluidas en el modelo predictivo de regresión logística binaria utilizando tuberculosis pulmonar como variable dependiente dicotómica.

\section{Consideraciones éticas}

En todo momento de la investigación se mantuvo la confidencialidad de la información. Se trabajó exclusivamente con datos epidemiológicos, determinantes específicos y de importancia para encontrar los factores de riesgo de tuberculosis pulmonar.

\section{RESULTADOS}

Anualmente, se reportan entre siete a diez casos de tuberculosis pulmonar. En el periodo de estudio se notificaron 96 casos en trabajadores de salud. En la figura 1 se observan las tasas de incidencia anual (por 100000 trabajadores de salud). La tasa habitual de tuberculosis fue 348 por 100000 trabajadores de salud.

El 53,12\% (51 de 96), de los trabajadores de salud afectados de TB fueron bacilíferos (BK: positivo), 8,30\% (8 de 96) presentaron tuberculosis multidrogoresistente (MDR), 3 casos se reportaron en el Servicio de Emergencia, 2 en Medicina, 2 en Pediatría y 1 en el Servicio de Cirugía. No existió diferencia estadísticamente significativa para adquirir TB-MDR según el servicio donde se labora (X2: $8,271, \mathrm{p}: 0,30)$. El tiempo promedio para el diagnóstico de tuberculosis, desde el inicio de los síntomas hasta el aislamiento del bacilo, fue 21,22 días ( $D S \pm 6,86$ ), con un valor mínimo de 14 y máximo de 31 días. El 52,10 \% (50 de 96), de TS estaban nombrados. La edad promedio del grupo de los casos fue de 35,52 años ( $D E \pm 10,22$ ), y en el grupo control fue 40,11 años ( $D E \pm 11,11)(p: 0,004)$. 


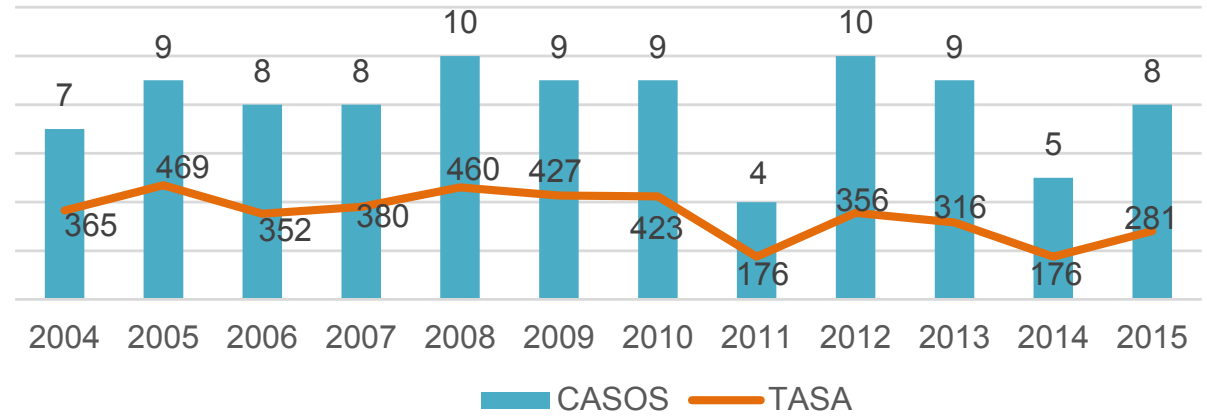

Figura 1. Tasa de incidencia de tuberculosis pulmonar en trabajadores de salud. HNDM. 2004-2015

La presencia de enfermedades concomitantes en los trabajadores se muestra en la figura 2 . Veinticuatro TS ( $25 \%$ ), en el grupo de casos, padecían de comorbilidades como diabetes mellitus (6), bronquitis crónica y asma (8), hipertensión (5), conectivopatía (4) y HIV (1). Contrariamente, en el grupo control 13 (13,5\%), padecían enfermedades similares al de grupo control, con gran prevalencia de hipertensión arterial (7 casos), pero con muy pocos casos de diabetes (2 casos), conectivopatía (1 caso), bronquitis crónica (2 casos) y malignidad (1 caso). Los TS con presencia de alguna comorbilidad tuvieron 2 veces más probabilidad de adquirir TB pulmonar que el grupo control.

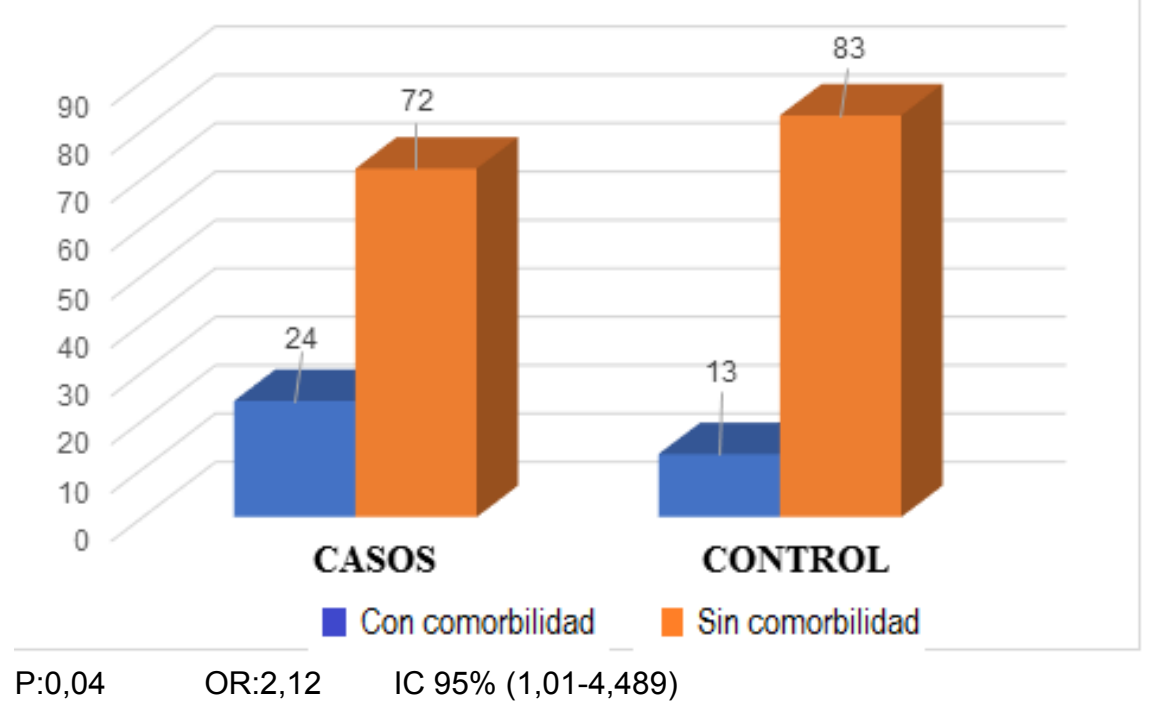

Figura 2. Riesgo de adquirir tuberculosis pulmonar en trabajadores de salud, según presencia de comorbilidad. HNDM.2004-2015

En la tabla 2 se observan los resultados del análisis univariado de las principales variables independientes que resultaron ser estadísticamente significativas: velocidad del flujo aéreo $\leq 0,7 \mathrm{~m}^{2} / \mathrm{s}$, presencia de aire acondicionado, hacinamiento medido por el área $\leq 2 \mathrm{~m}^{2} /$ persona, edad $\leq 40$ años, presencia de comorbilidad, y el tiempo de servicio $\leq 20$ años.

El género femenino tuvo discreta predominancia, tanto en el grupo de casos como en el grupo control ( $58,63 \%$ vs. 56,30 
$\%)$, sin alcanzar diferencia estadísticamente significativa (p: 0,07). El tiempo promedio de servicio laboral fue 9,23 años $(D E \pm 7,32)$ en el grupo de casos, y 16,57 años (DE \pm $12,16)$, en el grupo de controles; el $91,70 \%$ de los TS del grupo de casos estaban trabajando un tiempo $\leq$ de 20 años, versus el $64,60 \%$ en el grupo control. Los trabadores de salud con tiempo de servicio $\leq 20$ años tuvieron 6 veces más riesgo de adquirir la enfermedad, que los trabajadores con tiempo de servicio $\geq$ a 21 años, lo que fue estadísticamente significativo ( $p: 0,01)$.

Tabla 2. Factores de riesgo de tuberculosis pulmonar en trabajadores de salud, análisis univariado. HNDM. 2004-2015

\begin{tabular}{|c|c|c|c|c|c|c|c|c|}
\hline \multirow[t]{2}{*}{ Variables } & & \multicolumn{2}{|c|}{ Caso } & \multicolumn{2}{|c|}{ Control } & \multicolumn{3}{|c|}{ Estadísticos } \\
\hline & & Nro. & $(\%)$ & Nro. & $(\%)$ & $\mathbf{p}$ & OR & IC $95 \%$ \\
\hline \multirow[t]{2}{*}{$\begin{array}{l}\text { Ambiente } \\
\text { laboral }\end{array}$} & Medicina y Emergencia & 64 & $66,7 \%$ & 53 & $55,2 \%$ & 0,10 & 1,6 & $\begin{array}{l}0,90- \\
2,91\end{array}$ \\
\hline & Otros servicios & 32 & $33,3 \%$ & 43 & $44,8 \%$ & & & \\
\hline \multirow[t]{2}{*}{$\begin{array}{l}\text { Uso de respirador } \\
\mathrm{N}-95\end{array}$} & Poco frecuente & 63 & $65,6 \%$ & 52 & $54,2 \%$ & 0,10 & 1,6 & $\begin{array}{l}0,90- \\
2,80\end{array}$ \\
\hline & Frecuente & 33 & $34,4 \%$ & 44 & $45,8 \%$ & & & \\
\hline \multirow[t]{2}{*}{$\begin{array}{l}\text { Sistema de ventilación } \\
\text { cerrada }\end{array}$} & Ausente & 81 & $84,4 \%$ & 82 & $85,4 \%$ & 0,84 & 0,92 & $\begin{array}{c}0,40- \\
2,03\end{array}$ \\
\hline & Presente & 15 & $15,6 \%$ & 14 & $14,6 \%$ & & & \\
\hline \multirow[t]{2}{*}{$\begin{array}{l}\text { Patrón del flujo } \\
\text { aéreo }\end{array}$} & Turbulento & 61 & $63,5 \%$ & 56 & $58,3 \%$ & 0,46 & 1,2 & $\begin{array}{l}0,69- \\
2,22\end{array}$ \\
\hline & Laminar & 35 & $36,5 \%$ & 40 & $41,7 \%$ & & & \\
\hline \multirow[t]{2}{*}{$\begin{array}{l}\text { Velocidad de flujo } \\
\text { aéreo }(\mathrm{m} / \mathrm{s})\end{array}$} & $\leq 0,7 \mathrm{~m}^{2} / \mathrm{s}$ & 65 & $67,7 \%$ & 50 & $52,1 \%$ & 0,02 & 1,9 & $\begin{array}{l}1,07- \\
3,49\end{array}$ \\
\hline & $\geq 0,8 \mathrm{~m}^{2} / \mathrm{s}$ & 31 & $32,3 \%$ & 46 & $47,9 \%$ & & & \\
\hline \multirow[t]{2}{*}{$\begin{array}{l}\text { Dirección del flujo } \\
\text { aéreo }\end{array}$} & Inapropiado & 57 & $59,4 \%$ & 48 & $50,0 \%$ & 0,19 & 1,46 & $\begin{array}{l}0,82- \\
2,58\end{array}$ \\
\hline & Apropiado & 39 & $40,6 \%$ & 48 & $50,0 \%$ & & & \\
\hline \multirow[t]{2}{*}{$\begin{array}{l}\text { Luz } \\
\text { ultravioleta }\end{array}$} & NO & 84 & $87,5 \%$ & 81 & $84,4 \%$ & 0,53 & 1,2 & $\begin{array}{l}0,57- \\
2,93\end{array}$ \\
\hline & sí & 12 & $12,5 \%$ & 15 & $15,6 \%$ & & & \\
\hline \multirow[t]{2}{*}{ Aire acondicionado } & sí & 44 & $45,8 \%$ & 28 & $29,2 \%$ & 0,01 & 2,05 & $\begin{array}{l}1,13- \\
3,72\end{array}$ \\
\hline & NO & 52 & $54,2 \%$ & 68 & $70,8 \%$ & & & \\
\hline \multirow[t]{2}{*}{ Hacinamiento } & $\leq 2 \mathrm{~m}^{2 /}$ persona & 15 & $15,6 \%$ & 5 & $5,2 \%$ & 0,01 & 3,37 & $\begin{array}{l}1,17- \\
9,68\end{array}$ \\
\hline & $>2 \mathrm{~m}^{2} /$ persona & 81 & $84,4 \%$ & 91 & $94,8 \%$ & & & \\
\hline \multirow[t]{2}{*}{ Situación laboral } & Contratado & 46 & $47,9 \%$ & 35 & $36,5 \%$ & 0,10 & 1,6 & $\begin{array}{l}1,90- \\
2,58\end{array}$ \\
\hline & Nombrado & 50 & $52,1 \%$ & 61 & $63,5 \%$ & & & \\
\hline \multirow[t]{2}{*}{ Edad } & $\leq 40$ años & 68 & $70,8 \%$ & 52 & $54,2 \%$ & 0,01 & 2,05 & $\begin{array}{l}1,13- \\
3,72\end{array}$ \\
\hline & $>40$ años & 28 & $29,2 \%$ & 44 & $45,8 \%$ & & & \\
\hline \multirow[t]{2}{*}{ Género } & Femenino & 56 & $58,3 \%$ & 54 & $56,3 \%$ & 0,77 & 1,08 & $\begin{array}{c}0,51- \\
1,92\end{array}$ \\
\hline & Masculino & 40 & $41,7 \%$ & 42 & $43,8 \%$ & & & \\
\hline \multirow[t]{2}{*}{ Comorbilidad } & Presente & 24 & $25,0 \%$ & 13 & $13,5 \%$ & 0,04 & 2,12 & $\begin{array}{l}1.01- \\
4,48\end{array}$ \\
\hline & Ausente & 72 & $75,0 \%$ & 83 & $86,5 \%$ & & & \\
\hline \multirow[t]{2}{*}{ Tiempo de servicio } & $\leq 20$ años & 88 & $91,7 \%$ & 62 & $64,6 \%$ & 0,01 & 6,03 & $\begin{array}{c}2,61- \\
3,91\end{array}$ \\
\hline & $\geq 21$ años & 8 & $8,3 \%$ & 34 & $35,4 \%$ & & & \\
\hline Total & & 96 & $100,0 \%$ & 96 & $100,0 \%$ & & & \\
\hline
\end{tabular}


El 65,6\% (63 de 96) de los trabajadores de salud del grupo de casos no usaron la mascarilla frecuentemente, versus el 54,2 \% (52/96) en el grupo control: no se encontró diferencia significativa entre los grupos $(P: 0,10)$. En el análisis del riesgo de contraer tuberculosis, a través de regresión logística binaria, se encontró que los trabajadores que no usan respiradores con frecuencia tienen aproximadamente $50 \%$ más probabilidades de adquirir la enfermedad comparados, con el grupo que sí lo utilizaron con frecuencia (OR: 1,61. IC $95 \%$ : 0,90-2,89).

El $72,92 \%$, de los casos de tuberculosis pulmonar de los trabajadores se produjeron en áreas clínicas (medicina, emergencia y pediatría), de ellas, las dos primeras tuvieron carga alta de tuberculosis.

Las áreas quirúrgicas tuvieron riesgo intermedio, en estos servicios encontramos el 11,45\% de los casos. Finalmente, los servicios auxiliares (apoyo diagnóstico, mantenimiento, limpieza, administrativo, entre otros), resultaron tener un bajo riesgo (3,13\% de casos). El 66,67\% de los TS afectados por tuberculosis pulmonar correspondían a los departamentos de Medicina y Emergencia. El riesgo de contraer tuberculosis pulmonar en los TS que laboran en los servicios mencionados fue de 1,6 veces más que los trabajadores de otros departamentos hospitalarios.

La población $\leq$ de 40 años en el grupo de casos fue 68/96 versus 52/96 en el grupo control (Figura 3 ). Los TS con edad s de 40 años tuvieron mayor riesgo de padecer tuberculosis clínica que los trabajadores mayores de 40 años. En el grupo de casos, la proporción de edad $\leq 40$ / > de 40 años, fue de 2,42 mientras que, en el grupo control, la proporción fue de 1,18. Los TS con edad $\leq 40$ años tuvieron, aproximadamente, dos veces más probabilidad (OR:2,05), de contraer la enfermedad que los trabajadores mayores de 40 años.

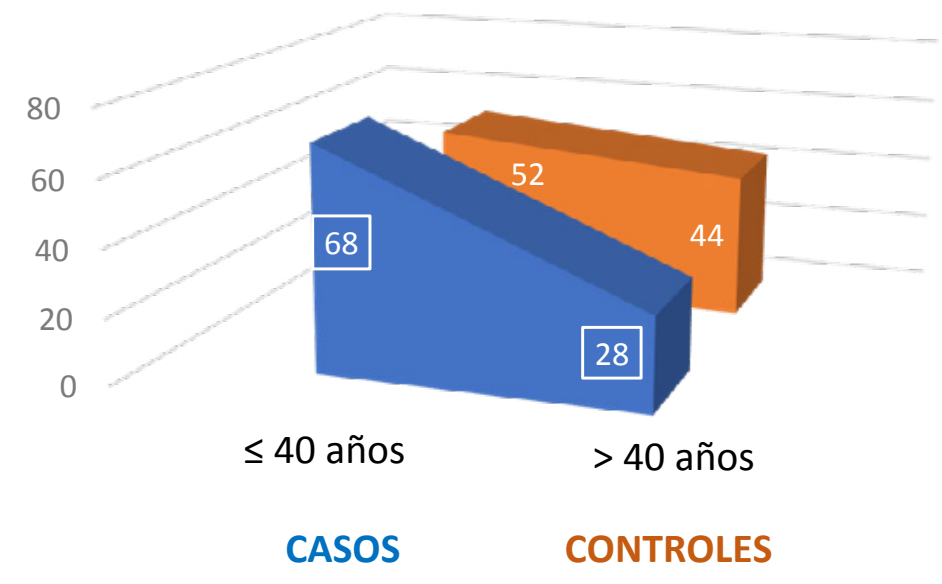

Figura 3. Riesgo de enfermar de tuberculosis pulmonar en trabajadores de salud, según edad. HNDM:2004-2015

El 15,60\% de TS en el grupo de estudio disponía de un área libre, menor o igual de $2 \mathrm{~m}^{2}$ /persona, comparado con el $5,20 \%$ en el grupo control. Se encontraron diferencias estadísticamente significativas entre el personal de salud que laboraba en ambientes con áreas libre $\leq \mathrm{a} 2 \mathrm{~m}^{2} /$ persona, comparados con los trabajadores que disponían de áreas mayores a $2 \mathrm{~m}^{2} /$ persona $(\mathrm{p}: 0,01)$. Los primeros tuvieron 3,3 veces más probabilidades de contraer tuberculosis, a diferencia de los trabajadores que disponían de área libre mayor a $2 \mathrm{~m}^{2} /$ persona.

Encontramos una velocidad promedio de flujo aéreo de 1,1 $\mathrm{m} / \mathrm{s}$ en los tópicos $A$ y $B$ del Servicio de Emergencia, en Medicina fue de 0,8 y en Neumología, de $1,4 \mathrm{~m} / \mathrm{s}$.

Los ambientes o servicios con velocidad de flujo aéreo menor o igual a $0,7 \mathrm{~m} / \mathrm{s}$, tuvieron riesgo incrementado de adquirir tuberculosis comparados con los ambientes o servicios que disponían de flujo aéreo mayor o igual a 0,8 m/s con OR:1,92 (IC95\%: 1,07-3,46), este hallazgo es estadísticamente significativo ( $p: 0,02)$. La disposición al flujo turbulento se encontró, especialmente, en las salas de medicina, hall de emergencia, tópicos A y $B$ de emergencia y pasadizos intrahospitalarios. 
Las variables significativas del análisis univariado, fueron analizadas con un modelo predictivo de regresión logística multivariado, en la tabla 3 podemos observar que la presencia de hacinamiento y el tiempo de servicio fueron factores de riesgo para adquirir tuberculosis, con una asociación estadística significativa $(\mathrm{p}<0,05)$.

Tabla 3. Factores de riesgo para tuberculosis pulmonar en trabajadores de salud, análisis de regresión logística multivariada. HNDM. 2004-2015

\begin{tabular}{|lcrrrrrrr|}
\hline VARIABLES & B & $\begin{array}{c}\text { Error } \\
\text { estándar }\end{array}$ & Wald & OR & P & \multicolumn{2}{c|}{ IC 95\% } \\
\hline Tiempo de servicio & 2,049 & 0,457 & 20,097 & 7,759 & 0,00 & 3,168 & 19,004 \\
Hacinamiento & 1,477 & 0,636 & 5,403 & 4,381 & 0,02 & 1,261 & 15,226 \\
\hline
\end{tabular}

\section{DISCUSIÓN}

La tuberculosis ocupa el décimo lugar como causa de hospitalización en el Hospital Nacional Dos de Mayo, representa el 1,89\% del total de las hospitalizaciones y es responsable del $11 \%$ de la mortalidad global en pacientes hospitalizados. El 84,54 \% del total de trabajadores, pertenece al personal del equipo asistencial (médicos, enfermeras, técnicos de enfermería, residentes e internos) y el 15,46 \% restante corresponde al personal administrativo, personal de servicio y personal de apoyo diagnóstico. Resultados que se relacionan directamente con el contacto cercano y frecuente del personal asistencial.

Según datos del Banco Mundial, la incidencia estimada de tuberculosis ha descendido en el Perú de 188 casos por cada 100000 habitantes en el año 2000, hasta 117 casos por 100000 para el $2016^{(1)}$. Pero esto no se ha reflejado en la incidencia de la tuberculosis nosocomial, especialmente en los TS, que siguen presentando tasas de incidencia de tuberculosis clínica que oscilan entre 176 a 469 por 100 000 trabajadores. La tasa habitual de incidencia de TB encontrada en TS del Hospital Nacional Dos de Mayo (348 por 100 000) fue, aproximadamente, 4,02 veces mayor que la tasa de incidencia nacional notificada el 2016, según reporte del Ministerio de Salud peruano (86,4 por 100 000). Los resultados encontrados en nuestra investigación fueron similares a los de otros hospitales nacionales en el mismo periodo. Según el reporte epidemiológico de tuberculosis del Ministerio de Salud Peruano del 2015, los hospitales públicos del Ministerio de Salud (MINSA), con mayor tasa de incidencia de TB clínica en TS para el 2013 fueron Cayetano Heredia (650 por 100 000), Arzobispo Loayza (400 por 100 000), Dos de Mayo (390 por 100 000), Hipólito Unanue (250 por 100 000) y Daniel A. Carrión del Callao (340 por 100 000).

El siguiente año, 2014, el Hospital Nacional Hipólito Unanue tuvo la mayor incidencia de TB activa del personal de salud (440 por 100 000), seguido del Hospital Nacional
Cayetano Heredia 330 por 100 000, el hospital Nacional Dos de Mayo (290 por 100 000), y en el cuarto lugar se ubicó el Hospital Sergio Bernales con 270 por 100000 trabajadores de salud ${ }^{(4)}$.

La mayor incidencia de tuberculosis de TS en los hospitales Cayetano Heredia (2013) e Hipólito Unanue (2014) se explica, probablemente, por los altos niveles de hacinamiento en sus instalaciones y escasa ventilación e iluminación, consecuentemente. En la infraestructura moderna del hospital Cayetano Heredia se observan techos bajos y ventanas pequeñas, lo que facilita la diseminación de la enfermedad. Respecto al hospital Hipólito Unanue, la alta incidencia se debe probablemente, a la población asignada a este nosocomio, que presenta una alta carga de tuberculosis.

Los hospitales antiguos, construidos antes de la década de los cincuenta, tienen ventanas y puertas grandes que maximizan la ventilación e iluminación natural y proveen mayor protección contra la infección de enfermedades aerotransportadas ${ }^{(5)}$. La mayoría de los casos de tuberculosis pulmonar se presentaron en técnicos de enfermería (23,96\%), médicos asistentes (19,76\%), residentes $(16,67 \%)$, e internos $(15,63 \%)$. Estos hallazgos, concuerdan con el reporte de Fica et al. ${ }^{(6)}$, que encontraron predominancia de este grupo ocupacional (técnicos de enfermería 35,7\%), y licenciadas de enfermería (14,3\%). Los técnicos de enfermería ocupan el primer o segundo lugar como TS más afectados en diferentes reportes publicados ${ }^{(7-9)}$.

Recientemente, se ha reportado que el grupo de técnicos de salud constituye el 66,6\% de todos los casos de tuberculosis en TS. Esta elevada incidencia en este grupo ocupacional se relaciona, probablemente, con su estilo de trabajo, ya que se encargan de administrar el tratamiento directamente observado y supervisado (DOTS), labor que les corresponde según la norma técnica. En los ambientes hospitalarios, los técnicos de enfermería trabajan en turnos rotatorios de 12 
horas, indudablemente, a más horas de trabajo y mayor contacto cercano con el paciente, las probabilidades de contagio son mayores. Además, las personas de este grupo son relativamente jóvenes, lo que se considera, en algunas investigaciones, como un factor de riesgo para contraer la enfermedad. Finalmente, la elevada posibilidad de que los técnicos y auxiliares de enfermería contraigan TB pulmonar en técnicos y auxiliares de enfermería se relacionaría probablemente, con su nivel socioeconómico, pues, la remuneración económica que perciben es la más baja de todo el equipo asistencial de salud. Estas características los convierten en una población importante de riesgo. La tasa incrementada de incidencia de tuberculosis en técnicos auxiliares ha sido reportada desde hace muchos años en diferentes investigaciones en todo el mundo $\mathrm{y}$, específicamente, en Latinoamérica ${ }^{(10-13)}$.

Diferentes investigaciones han reportado el empleo inadecuado 0 inexistente de la protección de barrera (respirador N. ${ }^{\circ}$ 95). Bullón $A$, en un estudio realizado en Chiclayo, Perú el 2017, reportó que solo el 63,3\% de los TS usaban el respirador con frecuencia, de ellos, solo el 56,7 \% lo utilizaba correctamente, a pesar de tener un nivel de conocimiento medio a alto de la utilidad e importancia de este ${ }^{(10)}$. La tasa de enfermedad TB de los técnicos de enfermería en el HNDM osciló entre 197 y 789 por 100000 trabajadores de salud, la menor tasa se alcanzó en 2008, y la más alta, en 2010. Diaz et al. en la investigación realizada en un hospital docente de Cuba encontraron que el riesgo de TB en personal técnico de enfermería era 8,21 veces mayor que el grupo de enfermeras ${ }^{(13)}$.

Los médicos del HNDM ocuparon el segundo lugar, con una tasa de incidencia que fluctúo desde cero (ningún caso en determinados años), hasta 1361 por 100000 en 2008. La tasa incrementada para ese periodo se relacionó con el traslado de la atención de emergencia a los ambientes pequeños de consultorios externos con la finalidad de ampliar y modificar las instalaciones del Servicio de Emergencia. Este, probablemente, fue el factor condicionante para el incremento de la incidencia de tuberculosis por encima de 1000 por 100 000. En un estudio de 5 años de seguimiento se encontraron 45 casos de TB pulmonar en el Hospital Nacional Dos de Mayo, en este periodo el grupo más afectado fue el de los médicos, especialmente, internistas $(21,40 \%)$, seguido de enfermeras (19\%) y médicos residentes (19\%), grupo asistencial, frecuentemente, encargados de la atención de salud en emergencia ${ }^{(14)}$. Estos hallazgos fueron similares a los encontrados por en China por Xiao-Ning $\mathrm{W}$ et al. que, mediante análisis multivariado, encontraron que ser enfermera presenta un riesgo relativo para TB pulmonar entre 1 a 8 veces mayor que el resto de los profesionales de la salud ${ }^{(15)}$.

La predominancia por el sexo femenino en los casos de tuberculosis en TS ha sido reportada en diferentes investigaciones ${ }^{(6,8,16,17)}$. En Chile, Fica et al., encontraron una predominancia significativa del género femenino $(78,6$ $\%)$, y Nakandakari et al., 58,9 \% (36 de 56). En nuestra investigación se encontró una discreta predominancia del género femenino $(58,30 \%)$, que no se consideró estadísticamente significativa $(p: 0,77)$. Estos hallazgos probablemente se relacionan a la a la mayor población hospitalaria femenina en el HNDM, especialmente, en el grupo de personal nombrado, donde la relación femenina/ masculino fue de 1,80. Estos hallazgos sugieren que, en nuestro hospital, la frecuencia de TB en el personal probablemente sea similar en ambos sexos.

Dentro del equipo de salud, los residentes e internos constituyen un grupo importante de riesgo para adquirir la enfermedad, por la modalidad de trabajo que realizan. Ellos son relativamente jóvenes, la gran mayoría contratados, y permanecen más de 8 horas al día en el hospital por periodos de 1 a 3 años (tiempo que dura su formación). De igual manera, están en contacto directo con los pacientes en la consulta ambulatoria o en emergencia, al realizar el examen clínico, o al redactar la historia clínica a la cabecera del paciente. Esta situación los convierte en un grupo especialmente vulnerable para adquirir tuberculosis activa.

Los médicos residentes y los internos del Hospital Dos de Mayo tienen las tasas más altas de TB pulmonar activa, se encontraron valores de hasta 1818 y 2727 por 100000 en ambos grupos ocupacionales. La tasa más alta en el grupo de los internos de medicina del HNDM fue en 2005 (2727 por 100 000), al igual que los médicos residentes en ese mismo periodo.

El 62,5\% (10 de 16) de los residentes afectados por tuberculosis estaban en el primer año del residentado (R-1), probablemente, porque son los que tienen mayor tiempo de contacto con los pacientes (abordan al paciente en un inicio, realizan historia clínica, el examen físico, anamnesis detallada y los procedimientos comunes, etc.). A medida que los residentes avanzan en su formación, tienen menor contacto con los pacientes (frecuentemente abordan al paciente para indicar el tratamiento o para el procedimiento final). Finalmente, la edad menor de 40 años de este grupo sería un factor de riesgo determinante para adquirir la enfermedad. Las tasas encontradas en nuestro estudio son superiores a las de Lamiado-Laborín et al., quienes reportaron en un hospital de México que los médicos residentes presentaban riesgo incrementado de infección y enfermedad clínica, confirmado por las diferencias estadísticas; se encontró una incidencia de 1846 casos por 100000 personas en los médicos residentes, cifra superior al de los médicos asistentes (860 por 100 000), de la misma institución ${ }^{(18)}$.

Doce enfermeras del Hospital Nacional Dos de Mayo (12,50\%), adquirieron la enfermedad, sin embargo, existen reportes 
contrarios en diferentes investigaciones, donde describen mayor riesgo de tuberculosis en personal de enfermería ${ }^{(18,19)}$. Este hallazgo, probablemente, se explicaría por el menor tiempo de contacto de las enfermeras con el paciente dentro de su labor rutinaria, comparados con los técnicos de salud, R-1 e internos. El contacto directo y sostenido como factores de riesgo independientes para contraer la tuberculosis, fue corroborado en diferentes estudios. La mayor tasa de incidencia de tuberculosis clínica registrada en las enfermeras en nuestra investigación fue 741 por 100 000 en 2007.

La mayoría de casos de TB activa se concentraron en los servicios de Medicina y Emergencia (66,67\%), probablemente, por la alta carga de la enfermedad en estos servicios, desconocimiento de la infección en un primer momento, el alto número de coinfección de $\mathrm{VIH}$ y tuberculosis en los pacientes que acuden a la consulta, demora en el diagnóstico, procedimientos urgentes como intubación oro o nasotraqueal, ventilación mecánica, ausencia de ambientes de aislados y el tiempo prolongado de permanencia de los pacientes. Todas estas condiciones convierten a los servicios de Medicina y Emergencia en vulnerables y contribuyen en gran magnitud en la trasmisión de la TB intrahospitalaria. Se encontró que el personal que labora en estos servicios en el HNDM tiene 1,6 veces más probabilidad de contraer la enfermedad activa, comparado con los trabajadores de otros servicios intrahospitalarios [OR: 1,6 (IC95 \% 0,90-2,91)]. Con altas tasas de infección de TB en emergencia es probable y susceptible que más trabajadores de salud presenten la enfermedad clínica. Escombe et al., encontró una tasa de incidencia de infección latente tuberculosa (ILT), en emergencia del HNDM de 1730 por 100000 trabajadores de salud ${ }^{(20)}$. A mayor población con ILT, es predecible mayor probabilidad de desarrollar la enfermedad clínica.

La edad del personal de salud con tuberculosis ha sido evaluada en diversas investigaciones, se reporta que la edad relativamente joven es un factor de riesgo asociado ${ }^{(21,22)}$. Se encontró que los trabajadores menores de 40 años tuvieron dos veces más probabilidad de adquirir la enfermedad clínica que los mayores de 40 años (p: 0,001). Este hallazgo, probablemente, se explica porque la población más afectada $(73,23 \%)$ fueron técnicos de enfermería, internos, residentes y enfermeras, quienes tienen edades relativamente menores, comparados con los médicos asistentes. La probabilidad de adquirir la TBP en el Hospital Dos de Mayo, según la edad fue el doble que lo encontrado por Riboty $(\mathrm{OR}: 1,1)$ en el Hospital Guillermo Almenara, ${ }^{(22)}$ probablemente, estas diferencias sean explicados por el tamaño de la población en los diferentes nosocomios.

Se han reportado que, a diferencia de la TB activa, la ILT se presenta con mayor frecuencia a edades relativamente mayores y a medida que se incrementa los años de trabajo
(15,23,24), la aparición de la enfermedad activa depende exclusivamente de la integridad del estado inmunológico del huésped, el contacto con pacientes bacilíferos y por las insuficientes medidas de prevención hospitalaria.

La labor en los TS es de 40 horas semanales en promedio. En la mayoría de ellos se produce inicialmente la infección latente, posteriormente, una minoría (10\%), pueden padecer la enfermedad clínica. En la investigación realizada en 2010 en el Servicio de Emergencia del Hospital Nacional Dos de Mayo, Escombe et al. encontró una prevalencia de $56 \%$, y una incidencia de 1730 por 100000 TS de ILT (infección latente tuberculosa). No se realizaron estudios de prevalencia e incidencia de tuberculosis activa.

En nuestra investigación, solo un tercio $(34,37 \%)$, de los trabajadores de salud, usaron los respiradores N95 en forma frecuente (casi siempre y siempre). Estos hallazgos difieren de los encontrados por Mirtskhulava et al. ${ }^{(25)}$, en Georgia EE. UU., quienes reportaron que el $60 \%$ de los TS, usaron respiradores al entrar en contacto con pacientes con TB; este hallazgo, posiblemente, está asociado a una mejor actitud preventiva de los TS norteamericanos, debido a que Georgia es uno de los estados con mayor carga de tuberculosis. Estos resultados contrastan con los encontrados por Barboza et al. (26), en Colombia, que reportan que solo el $21 \%$ de los trabajadores del Servicio de Emergencia usaron el respirador para la atención de pacientes. En este último estudio, el alto número de personal (79 \%) que no usaba la mascarilla N95, posiblemente, estuvo relacionado a que la investigación se realizó en un centro de primer nivel de atención, con escasa carga de tuberculosis, donde los trabajadores de salud, probablemente, descuidaron la protección personal.

Estos reportes sugieren que la actitud y conducta preventiva de los TS está directamente relacionada a la carga de tuberculosis de los establecimientos de salud. Mediante análisis univariado, se encontró que el riesgo de adquirir la enfermedad activa en TS que no usaron el respirador con la frecuencia adecuada es de 1,6 veces más alto que los trabajadores de salud que lo usaron frecuentemente. Resultados similares se encontraron en otros estudios donde se comprueba el uso infrecuente de los respiradores N95 en los trabajadores de salud ${ }^{(25-27)}$.

Esta deficiencia de los TS respecto al uso de los respiradores (investigada en diferentes épocas), apoya la hipótesis que los TS conocen la importancia de esta medida preventiva, pero que, en la práctica, solo una escasa proporción las cumplen. Esto es confirmado con las investigaciones de Rojas E, realizadas en Callao- Lima, quien concluye que el personal de enfermería de la red de salud estudiada presenta un nivel de conocimiento alto $(72 \%)$ acerca del rol preventivo de los respiradores, pero solo el 32 \% los cumple. Estas mismas investigaciones reportan que los trabajadores de salud no 
usaron la mascarilla con la frecuencia debida, e incluso en el grupo que los utilizaron, se detectaron errores en el empleo de los respiradores N95 ${ }^{(18,27)}$. Un programa efectivo de control de la TB requiere la identificación temprana de los casos infectantes, el aislamiento oportuno de los enfermos y tratamiento precoz y efectivo de los pacientes con TB activa. Indubitablemente, de la eficiencia de estas tres estrategias, dependerá la disminución de la incidencia de tuberculosis activa en el personal de salud ${ }^{(28)}$.

En nuestra investigación, la presencia de comorbilidad, (sobre todo asma bronquial, bronquitis crónica, uso prolongado de corticoterapia, hipertensión y diabetes mellitus), estuvo presente en 24 pacientes en el grupo de casos y en 13, en el grupo control. Los TS con una o más comorbilidades tuvieron una asociación estadísticamente significativa con el desarrollo de enfermedad tuberculosa activa (p:0,04). Los trabajadores de salud con alguna comorbilidad tienen el doble de riesgo de contraer tuberculosis pulmonar que los trabajadores sin comorbilidad [OR:2.12, IC $95 \%(1,01-4,48)]$. Un tercio $(33,34 \%)$ de los TS tuvieron enfermedad respiratoria (asma bronquial y bronquitis crónica). Debido a la naturaleza de la TB, enfermedad de transmisión aérea, y de localización preferente broncopulmonar, quienes no tienen “especialización” regional en la respuesta inmune (óptima actividad macrofágica), es probable que, cualquier proceso infeccioso, vulnere los mecanismos de defensa local, facilite la infección y, posteriormente, aparezca la enfermedad tuberculosa. Investigaciones en cobayos han demostrado las ventajas de la inmunidad de las mucosas en la protección contra infecciones micobacterianas ${ }^{(28,29)}$.

La Norma Técnica de Salud, Infraestructura y Equipamiento de los Establecimientos de Tercer Nivel de Atención (NT-119MINSA/DGIEM-V01-2015), establece los espacios mínimos necesarios para los diferentes ambientes. Así tenemos que, en las áreas destinadas a hospitalización de medicina y cirugía, el espacio es $12 \mathrm{~m}^{2}$ por cama, y $19 \mathrm{~m}^{2}$ para 2 camas; en hospitalización de pediatría (adolescentes) es $12 \mathrm{~m}^{2} /$ cama; y ginecología, de $15 \mathrm{~m}^{2}$ /cama. Se destaca además que los ambientes de hospitalización para aislados deben tener como mínimo un área de $18 \mathrm{~m}^{2} /$ cama. El área para atención ambulatoria entre 13,5 a $17 \mathrm{~m}^{2}$ entre otros. En nuestro estudio se encontró que las áreas de hospitalización de medicina tuvieron $10,55 \mathrm{~m}^{2} /$ cama, espacio inferior a la NT de nuestro país, y muy inferior a las recomendadas en países europeos como España (20 m²/cama).

Si se tiene en cuenta el aforo de espacios públicos (hospitales), y los espacios mínimos recomendados por la NT mencionada, una disminución del $50 \%$ del espacio útil/ $\mathrm{m}^{2} /$ persona, resulta un factor de riesgo para adquirir TB pulmonar. Los ambientes hacinados (áreas útiles $\leq 2$ $\mathrm{m} 2$ /persona), tuvieron 3 a 4 veces más riesgo de adquirir tuberculosis activa (p: 0,01).
El hacinamiento de los hospitales es un problema nacional que refleja crisis en los sistemas de salud, y que afecta directamente la calidad y el acceso a los servicios de salud, la solución requiere un abordaje urgente con participación multisectorial.

En 2012, la Contraloría General de la República realizó inspectorías a los hospitales del Ministerio de Salud (MINSA) y EsSalud (Seguro Social del Perú) de Lima Metropolitana y el Callao, donde, entre otros ambientes hospitalarios, evaluaron los servicios de emergencia. Según las normas técnicas de salud para los servicios de emergencia ( $\mathrm{RM} \mathrm{N}^{\circ}$ 386-2006/MINSA/NTS $N^{\circ}$ 042-MINSA) y la norma técnica para proyectos de arquitectura y equipamiento de las unidades de emergencia de establecimientos de salud (RM $\mathrm{N}^{\circ}$ 064-2001-SA/DM/), encontraron que todos los servicios de emergencia presentaban condiciones de hacinamiento. En todos los casos la demanda era superior a la capacidad de oferta. El $65 \%$ de los servicios de emergencia de 41 hospitales de EsSalud en todo el Perú, tienen camillas en los pasillos y esto ocurre en el $100 \%$ de los hospitales de II y III nivel de la capital. El 65,8 \% del personal de salud entrevistado consideró pequeños e insuficientes los ambientes destinados para la atención de emergencias. No se contaba con equipamiento suficiente para atender las emergencias en $52,3 \%$ de los casos.

Los nosocomios grandes de la capital fueron construidos cuando la población de Lima era de 5 a 6 millones de habitantes, actualmente, la población supera los 12 millones y los hospitales no han sido reestructurados acorde al incremento en la demanda. La OMS indica que los hospitales deben tener un periodo de vida de 30 años luego del cual, los hospitales deben ser reconstruidos y adecuados a la demanda y con tecnología actual.

En conclusión, un alto grado de hacinamiento y el tiempo de trabajo hospitalario menor a 20 años, son factores de riesgo para contraer tuberculosis pulmonar activa. Estos factores deben ser tomadas en cuenta en la elaboración de la infraestructura hospitalaria con estándares adecuados de bioingeniería ambiental y con monitoreo preventivo permanente de los trabajadores de salud, especialmente los adultos menores de 40 años.

\section{REFERENCIAS BIBLIOGRÁFICAS}

1. World Health Organization. Global Tuberculosis Report. Genova; 2017.

2. Chugh TD. Burden of nosocomial tuberculosis in healthcare workers in India. Current Med Res Practice. 2017; 7(1): 18-9.

3. Ministerio de Salud del Perú. Análisis de la situación de salud del Perú. Lima; 2013.

4. Ministerio de salud del Perú. Análisis de la situación epidemiológica de la tuberculosis en el Perú. Lima; 2015.

5. Escombe AR, Oeser CC, Gilman RH, Ñavincopa M, Ticona E, Pan W, et al. Natural ventilation for the prevention of airborne 
contagion. PLoS Med. 2007; 4(2): 309-16.

6. Fica CA, Ramonda CP, Jemenao MI, Zambrano GA, Cifuentes DM, Febré VN, et al. Tuberculosis in health care workers from a public health service in Santiago, Chile. Rev Chilena Infectol. 2009; 26(1): 34-8.

7. Llerena C, Zabaleta A. Evaluación por el laboratorio de los casos de tuberculosis en profesionales del área de la salud. Acta Med Colomb. 2014; 39(4): 321-6.

8. Nakandakari M, De la Rosa D, Gutiérrez J, Bryson W. Tuberculosis en trabajadores de salud: estudio epidemiológico y clínico en el Hospital Nacional Hipólito Unanue. Rev Med Hered. 2014; 25(3): 129-34.

9. Soto-Cabezas MG, Chávez-Pachas AM, Arrasco-Alegre JC, Yagui-Moscoso MJA. Tuberculosis en trabajadores de salud en el Perú 2013-2015. Rev Perú Med Exp Salud Publica. 2016; 33(4): 607-15.

10. Bullón Cuadra AC. Evaluación del uso de respiradores N95 en los trabajadores de salud como medida de control de transmisión de tuberculosis en la unidad especializada en tuberculosis y servicio de emergencia del Hospital Regional Docente Las Mercedes [Tesis]. Chiclayo: Universidad Particular San Martin de Porres. Facultad de Medicina Humana; 2017.

11. McKenna MT, Hutton M, Cauthen G, Onorato IM. The association between occupation and tuberculosis. A population-based survey. Am J Respir Crit Care Med. 1996; 154(3Pt.1): 587-93.

12. Naidoo S, Jinabhai CC. TB in health care workers in KwaZuluNatal, South Africa. Int J Tuberc Lung Dis. 2006; 10(6): 676-82.

13. Diaz Castrillo AO, Dueñas Mojena D, Lazo-Álvarez MA, BorrotoGutiérrez S, Gonzales-Ochoa E. Tuberculosis en trabajadores de salud un hospital psiquiátrico de La Habana. 1997-2003. Rev Panam Infectol. 2005; 7(3): 22-6.

14. Contreras CC, Lira VH. Tuberculosis in health workers, Dos de Mayo Teaching National Hospital. Lima, Peru. Int J Innov Stud Medical Sci. 2018; 2(2): 1-6.

15. Wang $X N$, He TL, Geng MJ, Song YD, Wang JC, Liu M, et al. Prevalence of and risk factors for tuberculosis among healthcare workers in chinese tuberculosis facilities. Infect Dis Poverty. 2018; 7(1): 26.

16. Mathew A, David T, Thomas K, Kuruvilla PJ, Balaji V, Jesudason $M$, et al. Risk factors for tuberculosis among health care workers in South India: a nested case-control study. J Clin Epidemiol. 2013; 66(1): 67-74

17. González C, Araujo G, Agoglia R, Hernández S, Seguel I, Sáenz C. Tuberculosis en trabajadores de salud. Medicina (Buenos Aires). 2010; 70: 23-30.

18. Lamiado-Laborín R, Cabrales-Vargas N. Tuberculosis in health care workers at a general hospital in Mexico. Infect Control Hosp Epidemiol. 2007; 27(5): 449-52.

19. Borroto Gutiérrez S, Sevy Court JI, Fumero Leru M, González Ochoa E, Machado Molina D. Riesgo de ocurrencia de la tuberculosis en los trabajadores del Hospital Universitario Neumológico Benéfico de La Habana. Rev Cubana Med Trop. 2012; 64(1): 55-60.

20. Escombe AR, Huaroto L, Ticona E, Burgos M, Sanchez I, Carrasco $L$, et al. Tuberculosis transmission risk and infection control in a hospital emergency department in Lima, Peru. Int J Tuberc Lung Dis. 2010; 14(9): 1120-6.

21. Tudor C, Van Der Walt ML, Margot B, Dorman SE, Pan WK, Yenokyan G, et al. Occupational risk factors for tuberculosis among healthcare workers in KwaZulu-Natal, South Africa. Clin Infect Dis. 2016; 62(Suppl. 3): S255-61.

22. Riboty Lara A. Factores de riesgo ocupacionales y no ocupacionales para enfermar de tuberculosis pulmonar en trabajadores de salud-HNGAl 1995-2000 [Tesis]. Lima: Universidad Nacional Mayor de San Marcos. Facultad de Medicina Humana; 2005.

23. Pai M, Kalantri S, Nath Aggarwal A, Menzies D, Blumberg HM. Nosocomial tuberculosis in India. Emerg Infect Dis. 2006; 12(9): 1311-8.

24. Soto-Cabezas MG, Munayco Escate CV, Chávez Herrera J, López Romero SL, Moore D. Prevalencia de infección tuberculosa latente en trabajadores de salud de establecimientos del primer nivel de atención. Lima, Perú. Rev Perú Med Exp Salud Publica. 2017; 34(4): 649-54.

25. Mirtskhulava V, Whitaker JA, Kipiani M, Harris DA, Tabagari N, Owen-Smith AA, et al. Determinants of tuberculosis infection control-related behaviors among healthcare workers in the country of Georgia. Infect Control Hosp Epidemiol. 2015; 36(5): 522-8.

26. Barboza A, Peña O, Valderrama-Aguirre A, Restrepo H. Factores de riesgo para tuberculosis en trabajadores de servicios de urgencias, en dos niveles de atención de salud. Rev Col Salud Ocupac. 2014; 4(2): 30-3.

27. Rojas Noel EE. Nivel de conocimiento y grado de cumplimiento de las medidas de bioseguridad en el uso de la protección personal aplicados por el personal de enfermería que labora en la estrategia nacional de control y prevención de la tuberculosis de una red de salud - Callao 2015 [Tesis]. Lima: Universidad Nacional Mayor de San Marcos. Facultad de Medicina Humana; 2015.

28. Goonetilleke NP, MCShane H, Hannan CM, Anderson RJ, Brookes RH, Hill AV. Enhanced immunogenicity and protective efficacy against Mycobacterium tuberculosis of bacille Calmette-Guérin vaccine using mucosal administration and boosting with a recombinant modified vaccinia virus Ankara. J Immunol. 2003; 171(3): 1602-9.

29. Chen L, Wang J, Zganiacz A, Xing Z. Single intranasal mucosal Mycobacterium bovis BCG vaccination confers improved protection compared to subcutaneous vaccination against pulmonary tuberculosis. Infect Immun. 2004; 72(1): 238-46.

Fuentes de financiamiento:

Este artículo ha sido financiado por el autor.

\section{Conflictos de interés:}

El autor declara no tener ningún conflicto de interés.

\section{Correspondencia:}

Carlos Walter Contreras Camarena

Dirección: Av. Colonial 3008. A-403. Lima 01, Perú.

Teléfono: 912543776

Correo electrónico: ccontrerashndm@hotmail.com

$$
\begin{aligned}
& \text { Recibido: } 25 \text { de febrero de } 2019 . \\
& \text { Evaluado: } 13 \text { de mayo de } 2019 . \\
& \text { Aprobado: } 23 \text { de junio de } 2019 .
\end{aligned}
$$

(c) La revista. Publicado por Universidad de San Martín de Porres, Perú. (c) ву Licencia de Creative Commons Artículo en acceso abierto bajo términos de Licencia Creative Commons Atribución 4.0 Internacional. (http://creativecommons.org/licenses/by/4.0/)

\section{ORCID iDs}

Carlos Walter Contreras Camarena https://orcid.org/0000-0002-7394-995X 